\title{
THE LONG ROAD HOME: DRIVING PERFORMANCE AND OCULAR MEASUREMENTS OF DROWSINESS FOLLOWING NIGHT SHIFT-WORK
}

\author{
William J. Horrey ${ }^{1}$, Yulan Liang ${ }^{1}$, Michael L. Lee ${ }^{2}$, Mark E. Howard ${ }^{3}$, Clare Anderson ${ }^{4}$, \\ Michael S. Shreeve ${ }^{2}$, Conor O’Brien ${ }^{2} \&$ Charles A. Czeisler ${ }^{2}$ \\ ${ }^{1}$ Liberty Mutual Research Institute of Safety, Hopkinton, Massachusetts, USA \\ ${ }^{2}$ Division of Sleep Medicine, Harvard Medical School, Boston, Massachusetts, USA \\ ${ }^{3}$ Institute for Breathing \& Sleep, Austin Health, Melbourne, Australia \\ ${ }^{4}$ Monash University, Victoria, Australia \\ Email: william.horrey@libertymutual.com
}

\begin{abstract}
Summary: Because time-of-day effects on sleepiness interact with duration of prior waking, the commute home following a night shift is an especially vulnerable time for night shift workers. The current study aimed to explore the impact of night shift work on critical driving events as well as to explore physiological indices leading up to these events. Sixteen healthy night shift workers (18-65 years) each participated in two 2-hour driving sessions in an instrumented vehicle on a driving track. A baseline driving session was conducted following a night of rest, while another session was conducted following a night of shift work. Objective physiological measurements of drowsiness were monitored and collected continuously throughout the drive session as well as different measures of driving performance. Following the night-shift, drivers had higher Johns Drowsiness Scores (based on ocular measures) and were more likely to experience lane excursion events and investigator-initiated braking events than following a night's rest. While they also reported increasing failures in lane keeping ability, the pattern was not always consistent with actual observed data. The implications for countermeasures are discussed.
\end{abstract}

Ten percent of workers-approximately 9.5 million people-in the US work night shift or rotating shift schedules (US Department of Labor, 2002). These workers often experience high levels of fatigue and are vulnerable to drowsy driving, especially during the commute home following a night shift, because time-of-day effects on sleepiness interact with duration of prior waking and they tend to sleep during the biological day, when sleep is often shorter and of lesser quality (e.g., Kunert et al., 2007; Gold et al., 1992). The current study aimed to explore the impact of night shift work on critical driving events during a post-shift drive as well as explore physiological indices of drowsiness.

Sixteen healthy night shift workers $(\underline{\mathrm{M}}=46.7$ yrs, 18-65 years) drove an instrumented vehicle on a closed loop driving track for two hours under two conditions: following a night of rest (postsleep) and following a night of shift work (post-night shift). We recorded various physiological measurements and driving performance, including eye movements and blinks (Optalert and iView x HED systems), electroencephalography (EEG), electrooculography (EOG; Vitaport), the rate of lane excursions, and frequency of critical driving events (where an investigator [blind to condition] intervened using an emergency passenger-side brake pedal). Drivers also completed several ratings scales of sleepiness and performance every 15-minutes. 
The Johns Drowsiness Score, derived from duration, velocity and amplitude of eyelid movements (Johns et al., 2008), indicated that drivers were more drowsy and the increase in drowsiness over the course of the session was more pronounced in the post-night shift condition than the post-sleep condition $(\mathrm{p}<.05)$. More critically, drivers experienced more than three times the rate of lane excursions in post-night shift than post-sleep drives $(0.68 / \mathrm{min}$ and 0.20 /min, respectively, $\mathrm{p}=.03$ ) and the post-night shift accounted for all of the emergency braking events in the study (6/16 drivers post-night shift versus 0/16 post-sleep, $\mathrm{p}<.01)$. Drivers reported greater difficulty in lane keeping following the night shift compared to the post-sleep session ( $\mathrm{p}=.001)$. Interestingly, these ratings corresponded well with objective measures of lane keeping performance in the post-sleep condition; however, not for drivers in the post-night shift condition-particularly towards the end of the driving session as the effects of fatigue became more pronounced.

It is disturbing that nearly $40 \%$ of the drivers in our sample were involved in critical driving events following a night shift. Unfortunately, drivers on an actual commute home do not have the safety benefit of a secondary brake pedal and an alert passenger. While drivers reported increasing failures in lane keeping ability, the pattern was not always consistent with actual observed data. This is an important outcome as those drivers that are more fatigued and hence at greater risk of a motor vehicle crashes also have a less realistic appraisal of their own abilities and performance (e.g., Horne \& Reyner, 1999).

\section{ACKNOWLEDGEMENTS}

The current study was supported in part by an Institute of Breathing and Sleep Research Grant and from a grant from the National Institutes of Health (NIH T32-Hl 7901-14).

\section{REFERENCES}

Gold, D.R., et al. (1992). Rotating shift work, sleep, and accidents related to sleepiness in hospital nurses. Am J Public Health, 82, 1011-1014.

Horne, J. \& Reyner, L. (1999). Vehicle accidents related to sleep: A review. Occupational and Environmental Medicine, 56, 289-294.

Johns, M.W., Chapman, R., Crowley, K., \& Tucker, A. (2008). A new method for assessing the risks of drowsiness while driving. Somnologie, 12, 66-74.

Kunert, K., King, M.L., \& Kolkhorst, F.W. (2007). Fatigue and sleep quality in nurses. Journal of Psychosocial Nursing and Mental Health Services, 45(8), 30-37. 